with documented HIV infection indicates high risk behaviours related to HIV acquisition and/or ongoing HIV transmission. Clinics serving patients with STI syndromes in Zimbabwe and countries with similar HIV/STI epidemiology are of continued importance in HIV diagnosis and prevention.

\section{P09.24 THE AETIOLOGY OF GENITAL ULCER DISEASE AND ASSOCIATION WITH HIV INFECTION IN ZIMBABWE}

${ }^{1} \mathrm{~A}$ Machiha*, ${ }^{1} \mathrm{O}$ Mugurungi, ${ }^{2} \mathrm{M}$ Tshimanga, ${ }^{3} \mathrm{P}$ Kilmarx, ${ }^{1} \mathrm{M}$ Mungati, ${ }^{1} \mathrm{~J}$ Nyakura, ${ }^{2} \mathrm{G}$ Shambira, ${ }^{3} \mathrm{E}$ Gonese, ${ }^{3} \mathrm{~A}$ Herman-Roloff, ${ }^{4} \mathrm{~V}$ Kupara, ${ }^{5} \mathrm{D}$ Lewis, ${ }^{6} \mathrm{H}$ Handsfield, ${ }^{7} \mathrm{C}$ Rietmeijer. ${ }^{1}$ Ministry of Health and Child Care, Harare, Zimbabwe; ${ }^{2}$ University of Zimbabwe, Department of Community Medicine, Harare, Zimbabwe; ${ }^{3}$ US Centers for Disease Control and Prevention, Harare, Zimbabwe; ${ }^{4}$ ZICHIRE, Harare, Zimbabwe; ${ }^{5}$ The University of Sydney, Western Sydney Sexual Health, Sydney, Australia; ${ }^{6}$ University of Washington, Seattle, USA; ${ }^{7}$ Rietmeijer Consulting, Denver, USA

\subsection{6/sextrans-2015-052270.408}

Background In many countries, sexually transmitted infections (STI) are treated syndromically. Thus, patients diagnosed with genital ulcer disease (GUD) in Zimbabwe receive antimicrobials that cover infections with Treponema pallidum (TP: benzathine penicillin), Haemophilus ducreyi (HD: erythromycin) and herpes simplex virus (HSV: acyclovir). However, periodic surveys into the aetiology of GUD are necessary to inform treatment guidelines.

Methods For this study, we recently completed enrollment of 200 patients with GUD at 6 clinics in Zimbabwe. To date, test procedures have been completed for patients enrolled at Harare clinics $(\mathrm{N}=70)$. Ulcer specimens were obtained for testing using a validated multiplex polymerase chain reaction assay (M-PCR, National Institute of Communicable Diseases, Johannesburg) for TP (primary syphilis), HD (chancroid), HSV (genital herpes) and Chlamydia trachomatis strains associated with lymphogranuloma venereum (CT-LGV). Blood samples were collected for HIV testing by a standard rapid HIV test algorithm (First Response ${ }^{\mathrm{TM}}$ followed by Alere HIV 1/2 ${ }^{\mathrm{TM}}$ ) and considered positive when reactive on both.

Results To date, M-PCR testing is complete for all 70 patients with GUD recruited from the Harare clinics (38 men and 32 women). Of these, 17 (24.2\%) were positive for HSV, 8 (11.4\%) were positive for TP, and 1 was positive for CT-LGV. No cases of chancroid were detected. The overall HIV positivity rate was 43.1\%. HIV rates were higher among patients with HSV $(62.5 \%, \mathrm{p}=0.07$, Chi Square Test) and TP $(87.5 \%, \mathrm{p}<0.01$, Fisher's Exact Test).

Conclusions Genital herpes was the most common cause of GUD in our survey, followed by primary syphilis. The association of HIV infection with HSV and TP suggests high risk for co-transmission; however some HSV ulcerations among HIVinfected patients may be the result of HSV reactivation among immunocompromised patients. Our study methods and data should be relevant for other countries using a syndromic management to STI control.
P09.25 DURATION OF SYPHILIS SYMPTOMS AT PRESENTATIONS IN MEN WHO HAVE SEX WITH MEN IN AUSTRALIA: ARE CURRENT PUBLIC HEALTH CAMPAIGNS EFFECTIVE?

1,2EPF Chow*, 'K Dutt, ' $\mathrm{G}$ Fehler, 'I Denham, 1,2 $\mathrm{MY}$ Chen, ${ }^{3} \mathrm{C}$ Batrouney, ${ }^{1} \mathrm{~J}$ Peel, 1,2TRH Read, ${ }^{1,2} \mathrm{CS}$ Bradshaw, ${ }^{1,2} \mathrm{CK}$ Fairley. 'Melbourne Sexual Health Centre, Alfred Health, Melbourne, Australia; ${ }^{2}$ Central Clinical School, Faculty of Medicine, Nursing and Health Sciences, Monash University, Melbourne, VIC, Australia; ${ }^{3}$ Victorian AIDS Council/Gay Men's Health Centre, Melbourne, Australia

\subsection{6/sextrans-2015-052270.409}

Introduction The rapid rise in syphilis has prompted a number of public health campaigns to assist men who have sex with (MSM) men recognise and present early with symptoms. This study aimed to investigate the temporal trend of the duration of self-report symptoms and titre of rapid plasma regain (RPR) among MSM with infectious syphilis in relation to these campaigns.

Methods 761 syphilis cases among MSM diagnosed at the Melbourne Sexual Health Centre (MSHC) from 2007-2013 were reviewed. Median and interquartile range (IQR) of duration of symptoms and RPR titres in each year were calculated.

Results The median duration of symptoms for MSM with primary and secondary syphilis were 9 days (IQR: 6-14) and 14 days (IQR: 7-30), respectively. The overall median titre of RPR in secondary syphilis (128; IQR: 64-256) was higher than in primary (4; IQR: 1-32) and early latent syphilis (32; IQR: 4-64). The median duration of symptoms for primary $(\mathrm{p}=0.11)$, secondary $(\mathrm{p}=0.24)$ and titre of RPR level for primary $(\mathrm{p}=$ $0.35)$, secondary $(\mathrm{p}=0.08)$, and early latent syphilis $(\mathrm{p}=0.85)$ did not change over time. A strong positive correlation was observed between duration of symptoms and RPR titre in both primary $(\mathrm{r}=0.36, \mathrm{p}<0.001)$ and secondary $(\mathrm{r}=0.16, \mathrm{p}=$ $0.05)$ syphilis.

Conclusion Public health campaigns were not associated with a significant shorter time from onset of symptoms to treatment. Either more effective campaigns or alternative strategies such as more frequent testing in MSM should be promoted to control the syphilis epidemic in Australia.

Disclosure of interest statement None.

\section{P09.26 TRENDS IN GONORRHOEA POSITIVITY BY NUCLEIC ACID AMPLIFICATION TESTING VERSUS CULTURE IN HETEROSEXUAL MEN IN VICTORIA, AUSTRALIA, 2007-2014}

${ }^{1,2} \mathrm{PK}$ Mannion, ${ }^{1,2} \mathrm{CK}$ Fairley, ${ }^{2} \mathrm{G}$ Fehler, ${ }^{1,2 \mathrm{MY}}$ Chen, ${ }^{1,2} \mathrm{CS}$ Bradshaw, ${ }^{1,2}$ EPF Chow ${ }^{*}$. ${ }^{1}$ Central Clinical School, Faculty of Medicine, Nursing and Health Sciences, Monash University, Melbourne, VIC, Australia; ${ }^{2}$ Melbourne Sexual Health Centre, Alfred Health, Melbourne, VIC, Australia

\subsection{6/sextrans-2015-052270.410}

Introduction A three-fold increase in Neisseria gonorrhoeae (gonorrhoea) notifications was reported amongst low prevalence 
women without a change in the proportion positive by culture, suggesting that screening for gonorrhoea by nucleic acid amplification test (NAAT) is not recommended in low prevalence populations due to low specificity of detection. This study aimed to identify whether there is a change in the rates of gonorrhoea positivity by culture in heterosexual men in Victoria, consistent with increased notifications.

Methods Three data sources from 2007 to 2014 were used: notification data from the Victorian Department of Health, Medicare Item numbers for single and duplex chlamydia NAATs, and the proportion of new patients attending Melbourne Sexual Health Centre (MSHC) with gonorrhoea diagnosed by culture. Multivariate logistic regression analyses were used to examine the association between gonorrhoea positivity and known risk factors.

Results Gonorrhoea notifications by NAAT in heterosexual men in Victoria increased from 38 in 2007 to 169 in 2014. There has been a five-fold increase in annual Medicare Items for chlamydia and a second NAAT, whilst only a modest increase in chlamydia only NAAT. A total of 28,014 heterosexual men attended MSHC for first consultation between 2007 and 2014, and the overall gonorrhoea positivity by culture at MSHC was $0.93 \%(\mathrm{n}=260)$. No significant trend in annual gonorrhoea positivity (aOR 1.04, 95\% CI: $0.97-1.10, p=0.270$ ) was observed after adjusting for age, number of female sexual partners and condom use in the last three months, recent arrival in Australia, and previous sex overseas.

Conclusion These data suggest gonorrhoea positivity in heterosexual men is stable, despite increasing notification rates. An increase in false positive results due to low specificity of gonorrhoea NAAT may explain the observed trend.

Disclosure of interest statement This work was supported by the National Health and Medical Research Council (NHMRC) program grant APP1071269. No funding was received from pharmaceutical or biotechnology sectors.

\section{P09.27 EPIDEMIOLOGY OF GONORRHOEA IN CHINA FROM 2000 TO 2014}

XD Gong, J Jiang, XL Yue, F Teng, PX Men. Institute of Dermatology, Chinese Academy of Medical Sciences \& Peking Union Medical College, National Center for STD Control

\subsection{6/sextrans-2015-052270.411}

Introduction Since 1980s, sexually transmitted diseases (STD) reappeared in mainland China and the reported cases increased over years, the Ministry of Public Health set up national STD surveillance system to monitor trends. The results of surveillance showed that gonorrhoea was a prevalent STD and became an important public health issue. However, since 2000, incidence of gonorrhoea had decreased. In order to understand trends and epidemiological features of gonorrhoea recent 14 years in China, we conduct the analysis of data from the national system.

Methods Through 2000 to 2014, gonorrhoea case-reporting data collected from 31 provinces, autonomous regions and municipalities in China were analysed with descriptive epidemiological methods.

Results During 2000-2014, the trends of reported gonorrhoea incidence appeared declined totally, from 22.92 per 100,000 population in 2000 to 7.25 per 100,000 population in 2014 , with the annual average decrease of $7.89 \%$. There was a significant difference in gonorrhoea incidence in different regions. High incidence regions were Yangtze River delta (Zhejiang,
Shanghai, Jiangsu), Zhujiang River delta (Guangdong, Guangxi, Hainan), and then the northwest minority areas (Xinjiang, Ningxia, Inner Mongolia). The gonorrhoea incidences in male were higher than that in female, and the sex ratio of male to female has been increasing, from $1.96 \square 1$ in 2000 to $4.52 \square 1$ in 2014. The incidence of gonorrhoea in $20-44$ aged sex active population was peak among all age groups, maximum in 25-29 agegroup for male and female, and it presented decreased in all age groups except 15-19 age-group of male with increase of $4.18 \%$. Among 20 kinds of occupations, the proportion of the reported gonorrhoea cases for peasant workers was highest, accounting for $26.00 \%$, and the gonorrhoea incidences in all occupation groups were downtrend.

Conclusion Gonorrhoea remained being a major issue of public heath in China, the preventive measures should be effectively tailored in the light of gonorrhoea epidemiological features.

Disclosure of interest statement No potential conflicts of interest.

\section{P09.28 SURVEY ON THE DATA QUALITY OF SYPHILIS CASES REPORTED IN 5 PROVINCES OF CHINA}

XD Gong, XL Yue, N Jiang. Institute of Dermatology, Chinese Academy of Medical Sciences \& Peking Union Medical College, National Center for STD Control

\subsection{6/sextrans-2015-052270.412}

Introduction The results of syphilis surveillance showed that there were some singnicant problems in syphilis stage diagnosis and case-reporting in China. In order to assess the data quality of syphilis case reported in the country, and identify the problems for improving the quality, we conducted this sampling survey.

Methods According to the national protocol and syphilis diagnostic criteria, checking data with original reporting cards, clinical and laboratory logs of syphilis cases reported, assessment of diagnosis correctness were conducted by related experts at 43 medical hospitals/institutions sampled in 13 counties of 10 prefectures in 5 provinces based on geographic distribution to evaluate the data quality, and Excel 2003 and SPSS18.0 software were employed in data analysis.

Results The proportions of the reporting cards timely reported, completely filled, correctly filled, correctly entered by web for syphilis cases were as high as with 99.82\% (1691/1694), 97.05\% (1644/1694), 96.22\% (1630/1694) and 96.22\% (1630/1694), respectively, and the one of the reporting cards repeatedly reported was very low with $1.42 \%(24 / 1694)$ in 5 provinces. The proportions of correct diagnosis for syphilis cases was high with 95.40\% (2491/2611), however, the ones of correct stage for primary, secondary and latent syphilis were not high with $42.49 \%$ (167/393), 77.46\% (275/355) and 85.89\% (1497/1743), respectively. There was the severe bias of misclassification in primary and secondary syphilis, the reason of which was that some latent syphilis cases were falsely classified into primary and secondary ones by non dermatological/STD and in-patients department doctors.

Conclusion The indicators of data quality related to reporting information and correct diagnosis of syphilis cases were high, however proportion of correct stage for primary and secondary syphilis cases was low, which directly affected on analysis of syphilis incidence. It is urged that the training on related doctors in syphilis diagnosis criteria should be strengthened to substantially improve the data quality. 